\title{
The effects of stimulus density on orientation specific aftereffects of color adaptation
}

LEON W. TEFT AND FREEMAN T. CLARK ${ }^{1}$, DEPARTMENT OF PSYCHOLOGY, UNIVERSITY OF BRIDGEPORT, Bridgeport, Conn. 06602

Chromatic aftereffects to test gratings are affected significantly by differences in adaptation and test stimulus densities, i.e., number of edges per unit area. The strength of the aftereffect, as measured by the degree of angular disparity between adaptation and test grating orientation at which the aftereffect is lost, is a systematic function of adaptation and test density similarity.

Recent neurophysiological evidence points to the existence of edge detector mechanisms in the visual systems of lower organisms (Hubel, 1963; Hubel \& Weisel, 1962; and Mackay, 1961). These mechanisms are maximally responsive to stimuli presented in specific planar orientations, e.g., horizontal. Support for similar mechanisms in humans comes from the work of McCollough (1965), in which she demonstrates color aftereffects that are dependent upon line orientation in adaptation and test fields. In discussing these findings, McCollough suggests an explanation based upon color adaptation of orientation specific edge detectors. One prominent deficiency in research into human edge detector functioning is the lack of an objective measure of the aftereffect with systematic variations in test stimulus orientation and density, i.e., number of edges per unit area.

The purpose of the present experiment is to explore the effects on color aftereffects of varying density relations among adaptation and test stimuli using as a measure of these effects the degree of angular rotation of test orientation that can take place before the aftereffect disappears.

Method. Three university students with normal color and acuity vision served as $S s$ in this experiment.

Adaptation stimuli were presented in a horizontal or vertical orientation. Each adaptation orientation consisted of one of three densities, i.e., 7, 10, and 26 lines, projected on a 24 in. circular white field with a Lafayette projection tachistoscope. The horizontal gratings were paired with a red narrow bandpass filter with peak transmittivity at 700 millimicrons. The vertical gratings were paired with a Kodak Wratten No. 61 green filter with a peak transmittivity at 533.8 millimicrons. Following 5 min of dark adaptation, the $S$ was presented with $4 \mathrm{~min}$ of alternating red-horizontal and green-vertical gratings of one of the three densities. The $S$ was then presented with one of the three achromatic test stimulus densities in one of the two orientations. The test stimuli were identical in density to the adaptation stimuli with the exception of two lines enclosing a white bar at right angles to the grating and bisecting the center of the display. This pattern was chosen after pilot work indicated that it yielded the maximal aftereffect. The entire grating was then rotated slowly from the adaptation orientation until the $\mathrm{S}$ reported the disappearance of the aftereffect. The data were recorded in degrees of deviation from the adaptation orientation. The treatment combinations, i.e., adaptation orientation and density, test orientation and density, and clockwise or counterclockwise direction of rotation, were presented in a random order for each $\mathrm{S}$.

Results. The data were analyzed in a dual analysis of variance. For the horizontal test conditions, the interaction of adaptation and test stimulus densities proved to be significant beyond the .01 level $(F=7.40 ; \mathrm{df}=4 / 26)$. The means for this analysis are presented in Fig. 1. An inspection of these curves shows that for all adaptation and test stimulus density combinations maximal rotation of test orientation prior to loss of the color aftereffect occurs when the two stimulus densities are identical. As the test pattern deviates in density from the adaptation pattern density, less rotation from the adaptation orientation is possible before the negative aftereffect is lost. Conversely, under conditions in which there is maximal disparity between adaptation and test densities minimal rotation of the test grating away from the adaptation orientation will result in Ss reporting the loss of the chromatic aftereffect.

Similar results were obtained for the analysis of Ss' responses for vertical test conditions. The interaction of adaptation and test densities in this analysis yielded significance beyond the .01 level $(F=11.11 ; \mathrm{df}=4 / 26)$. These curves, highly similar to those obtained in the first analysis, indicate clearly the effects of disparity in stimulus pattern density on loss of color aftereffects under conditions of orientation rotation (cf. Fig. 2). For example, Ss adapting to fine chromatic gratings show maximal resistance to rotational loss of the aftereffect when the test stimulus is also fine in density.

Discussion. The results demonstrate clearly the effects of the density relations among adaptation and test stimulus gratings on the maintenance of color aftereffects. More specifically, test gratings that are identical in density, or number of edges per unit area, to adaptation gratings result in aftereffects that are less dependent upon grating orientation. As the test grating density departs from adaptation density the aftereffect becomes increasingly more dependent upon test stimulus orientation. The present experiment offers evidence that the orientation specific edge detectors presumed to mediate the aftereffect are sensitive to both orientation and density relations in adaptation and test patterns. As the density relation among the two stimulus patterns approaches identity the orientation specific receptors become

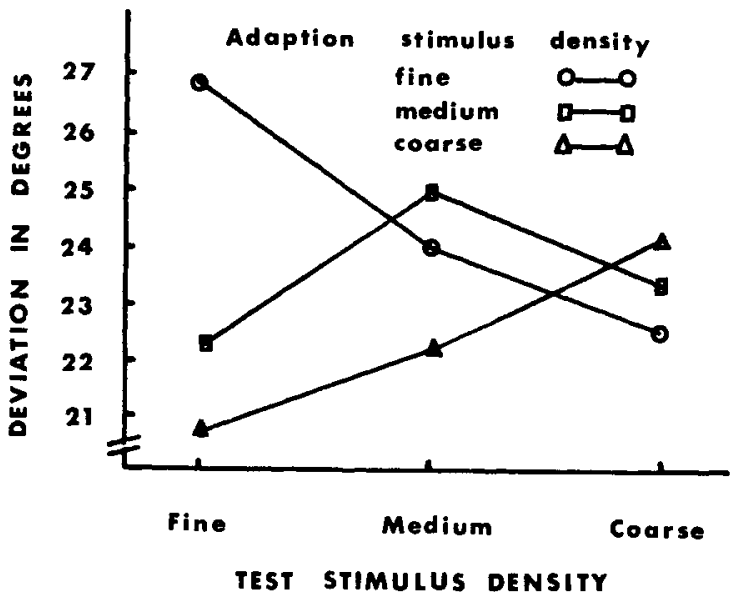

Fig. 1. Mean deviation in degrees at which aftereffect disappears for horizontal conditions.

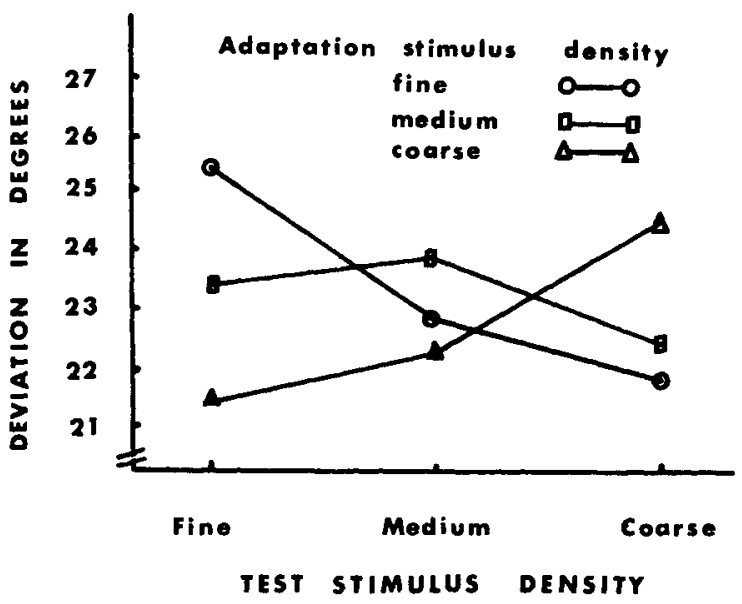

Fig. 2. Mean deviation in degrees at which the aftereffect disappears for vertical conditions. 
responsive to an increasing range of angular orientations. Conversely, as the density relation among adaptation and test patterns becomes increasingly dissimilar the orientation specific receptors are responsive to a narrowing range of stimulus orientation.

\section{REFERENCES}

HUBEL, D. H. Integrative processes in central visual pathways of the cat. $J$. Opt. Soc. Amer., 1963, 53, 58-66.

HUBEL, D. H., \& WIESEL, T. N. Receptive fields, binocular interaction, and functional architecture in the cat's visual cortex. J. Physiol, 1962, 160, $106-154$.

MACKAY, D. M. Interactive processes in visual perception. In W. A. Rosenblith (Ed.), Sensory communication. Cambridge, Mass.: MIT Press, 1961. Pp. 339-355.

McCOLLOUGH, C. Color adaptation of edge-dectors in the human visual system. Science, 1965, 149, 1115-1116.

NOTE

1. Now at the University of Michigan. 